International Journal of Engineering \& Technology, $7(2.12)(2018) 321-324$
International Journal of Engineering \& Technology
Website: $w$ ww.sciencepubco.com/index.php/IJET
Research Paper

\title{
A Cloud height observing system using high resolution whole sky image
}

\author{
Han-Kyung Yun ${ }^{1}{ }^{*}$, Sun-Min Whang ${ }^{2}$ \\ ${ }^{1}$ School of Computer Science and Engineering Korea University of Technology and Education, \\ ChonansiChungnam, 31253, South Korea \\ ${ }^{2}$ Research Center, I\&I Co. Ltd., ChungjoosiChungbook, 28788, South Korea
}

\begin{abstract}
Several methods were known to observe the cloud height. Since the meteorological administration mainly has been using human eye method, an automatic cloud height observer is proposed to reduce human errors. The proposed cloud observer system is for the cloud automatic observation system which has been operating at weathers stations in Korea since 1993. The high hand camera is adapted to get high resolution images without a capture board that has the low resolution. And the man-made passive filter for extracting sky reign is replaced by the active filter using image processing. The famous BRISK matching is selected instead of the block mating to get more precise disparity form a pair of stereo image.The Cloud cover announced by the Korean Meteorological Agency is the eye observation result nowadays, even though an intelligent cloud observation system was supplied. The reason is the impermissible error in the observed data of the automatic observation system. The current system is applied a man-made passive filter has to be changed periodically due to the boundary transition, but the proposed system is maintenance-free, since the active filter using edge detection algorithm automatically adjusts itself. The measuring of disparity which is important to analyze spatial information, has improved up to the resolution of observing height is $100 \mathrm{~m}$ by getting image data directly form the high resolution camera, the old resolution from the capture board. Also, the disparity analyzing method is changed to the BRISK matching algorithm instead of the block mating algorism to get more deliberate disparity analysis since the dimension of the block is too big relatively compare to the cloud in the stereo all sky image. The test results are acceptable range which is human observation value $\pm 10 \%$ since the resolution of observing height is $100 \mathrm{~m}$ at lower cloud in the sky. The proposed system is conducting the comparison research to compare with the result of reading satellite images and ceilometer.
\end{abstract}

Keywords: BRISK Matching; Cloud Height; Image Processing; Stereo Vision; Whole Sky Images; SWARD.

\section{Introduction}

The cloud amount affects meteorological variables as well as weather forecast. Especially lower clouds affect local weather and it is well known that one of main factors to induce a severe bad weather locally. Also, it is an absolute variable for determining solar radiation and sunshine on ground. Local weather information is able to be highly used in an industry using solar energy including farming industry and air traffic as well as airports. However, cloud amount lacks an objective evaluation due to visual observation on the ground at present, which has low spatiotemporal resolution. To overcome the limitations of bare-eye observation, satellite data are employed in calculating the cloud amount but they are not identical to those measured from ground and there is still a problem to get objective data. Another helpful measuring device (Allmen\&Kegelmeyer, 1997, Neto et el, 2010, Kazantzidis et el, 2012), Ceilometer (Costa-Surós et el, 2016)is one of devices to measure of cloud-height, but the device has a problem since it can only measure the height of cloud in the direction the vertical direction of the device. Hence the cloud is generally observed or measured by human-eyes every hour about 50 local meteorological agencies in Korea. Automation of observing cloud has been issuing since 2009. Because thecurrent used measuring system was developed at the same time, the system should be improved by applying rapidly developed the devices and computing technolo- gies to meet people's expectation. This study is the second stages which is the observing cloud-height. The first stage was the measuring of cloud-amount and the observing system was developed at that time, and WSI (Whole Sky Imager) or TSI (Total Sky Imager) were invited to improve the calculation of the cloud amount. Also few studies related to it recently have been conducted domestically. The previous our study developed algorithm of calculating the effective sky zone to get the cloud amount from the whole sky image data taken the automatic cloud observation system as shown in Fig. 1 and the table which had been supplied to the Daikwan-ryoung local metrological agency in Korea. The difference between two systems also shows in table 1 . To evaluate the performance of the proposed system, comparison was made with the current used method and with the eye observation, since the result of the old method has not given reliable data compared naked eye observation. The result is shown that the proposed method has more reliable and accurate. Finally, the observing data using our proposed system has been approved by the agency. The agency is doing more detailed analysis, seasonable, and a monthly analysis are required to supply other remaining local metrological agencies. The goal of this study is a developing preprocessing step to get the disparity of a pair of images to get the cloud-height. 

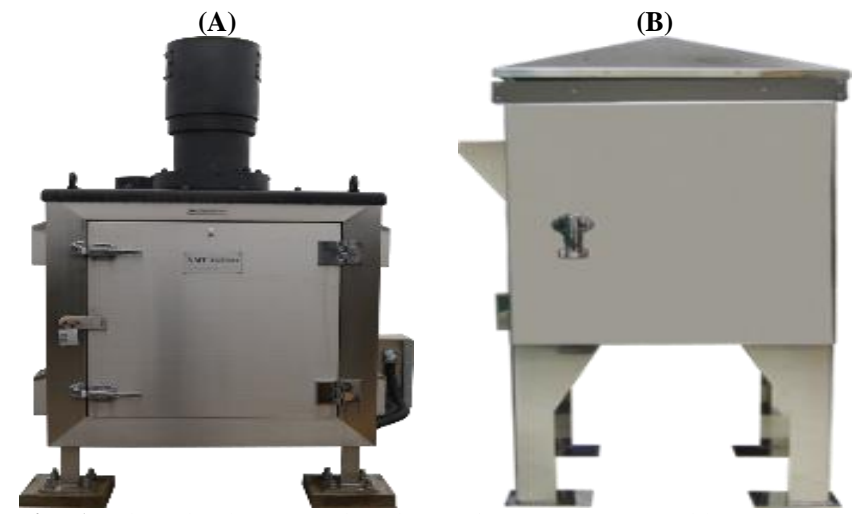

Fig. 1: The Cloud Automatic Observation System (A) Old System (B) Proposed System.

Table 1: Comparisons between Old System and Improved System.

\begin{tabular}{|c|c|c|}
\hline & old system & improved system \\
\hline Package & $\begin{array}{l}\text { Image acquisition } \\
\text { parts exposed }\end{array}$ & $\begin{array}{l}\text { all devices under cover due to } \\
\text { rain, snow and dust }\end{array}$ \\
\hline $\begin{array}{l}\text { Image } \\
\text { acquisition } \\
\text { device }\end{array}$ & $\begin{array}{l}\text { Video Camera: } \\
\text { Smsung SCH-745 }\end{array}$ & $\begin{array}{l}\text { DSLR,CannonEOS 100D with } \\
\text { EF 8 15mm 1:1.4L USM } \\
\text { fisheye lens }\end{array}$ \\
\hline Capture board & Used(640X480) & Not used \\
\hline $\begin{array}{l}\text { Image } \\
\text { resolution }\end{array}$ & $640 \times 480$ & $5,184 X 3,456$ \\
\hline $\begin{array}{l}\text { Comm. } \\
\text { protocol }\end{array}$ & RS-232, RS-422 & TCP/IP \\
\hline
\end{tabular}

\section{Fisheye camera calibration and image cor- rection}

Image of the cloud automatic observing system

The cloud automatic observing system for the cloud height was installed in Daejeon local metrological agency in April. The distance of two cameras is $36 \mathrm{~m}$ with the parallel axis. Because the equipped lens is a fisheye, images are whole sky image as shown in Fig. 2 but the top and button regions of image are corrupt since the camera is not a full frame camera and the corrupt ratio is 1.5 for our case. The angle of filed is 140 degrees of the vertical axis, and 180 degrees of the horizontal axis. Those are satisfied by the metrological agency since that field of view is enough to observing a local weather.

The camera with the fisheye lens was calibrated using a chessboard with $5 \mathrm{X} 5 \mathrm{Cm}$ grid and the calibrated image is shown in Fig. 3. The top picture was corrected using Ying's SWARD camera calibration toolbox (Ying et el, 2014)to examine our system. The result shows in Table 2. The camera distortion center for $\mathrm{x}$ is 2558.72 pixels and the camera distortion center for $\mathrm{y}$ is 1732.68 pixels. The distortion parameter $\mathrm{k}_{1}$ is $-1.9 \times 10-7$ that can be ignored. The data of result of compared with the specification of camera in the proposed system. Since the CCD sensor of camera $5,184 \times 3,456$ pixels, its physical center is $2,592 \times 1,728$ and the average of error rate is only $0.78 \%$.

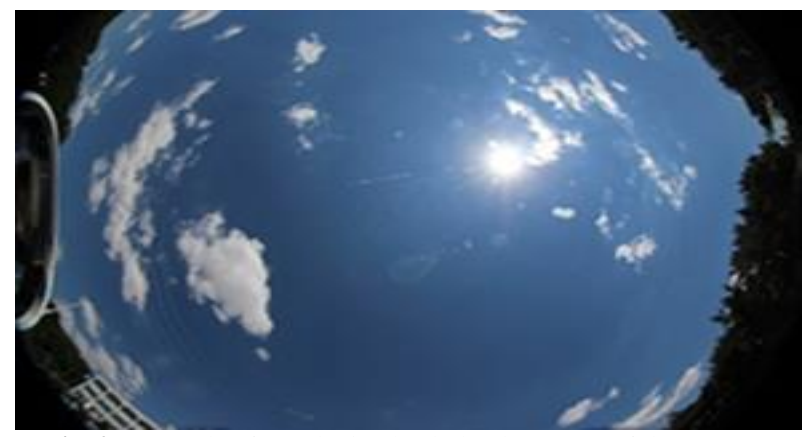

Fig. 2: Example of an Obtained Whole Sky Image of the System.

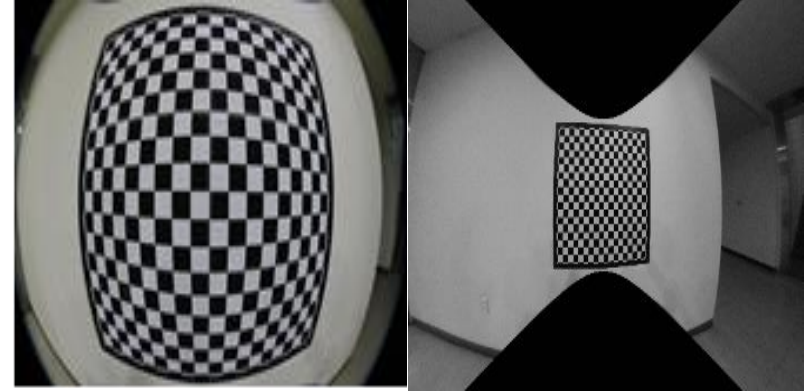

Fig. 3: A Result Image of Camera Calibration Using SWARD.

Table 2: Intrinsic Parameter of Camera Using SWARD Toolkit

\begin{tabular}{ll}
\hline Camera distortion center (center $\mathrm{x})$ & 2558.72 \\
\hline Camera distortion center (center $\mathrm{y})$ & 1732.68 \\
Distortion parameter $\left(\mathrm{k}_{1}\right)$ & $-1.9 \mathrm{X10}^{-7}$ \\
Camera focus length(f) & 5990.57 \\
\hline
\end{tabular}

Camera focus length(f)

Pinhole camera model and intrinsic matrix

Real 3D space is projected to 2D CCD sensor through the camera lens. The pinhole camera model can explain 3D to 2D perspective projection. If an object's center is $\mathrm{p}\left(\mathrm{x}_{\mathrm{u}}, \mathrm{y}_{\mathrm{u}}\right)$, the center of the image is $\mathrm{p}^{\prime}\left(\mathrm{x}_{\mathrm{c}}, \mathrm{y}_{\mathrm{c}}\right)$ on CCD in the pinhole camera model(Moldovan $\&$ Wada, 2004, Sturm, 2016) as shown in Fig.4. The lens of the camera is considered as the pinhole.

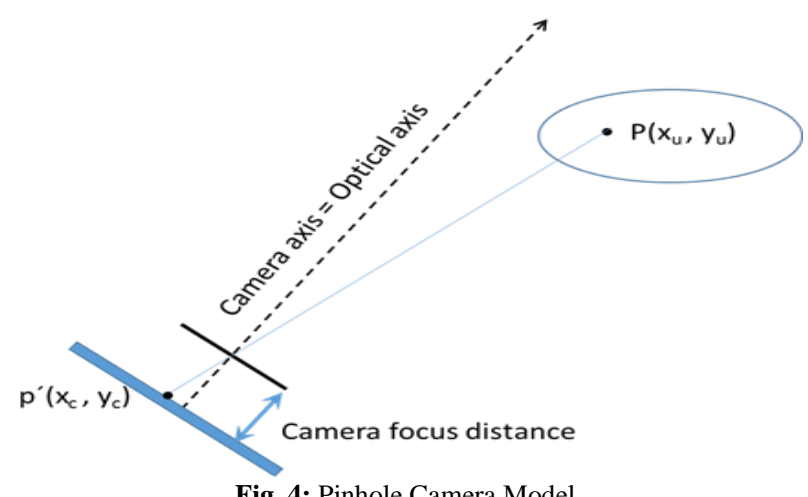

The image on CCD is distorted due to the fisheye lens. To observe cloud-height by a binocular disparity method, the distortion phenomena has to be corrected. The relation between 3D in the world coordinate and 2D in the image coordinate can be explained by camera internal parameters that are obtained during the calibration procedure. Those parameters are, camera focal lengths $\left(f_{x}, f_{y}\right)$, principal point or distortion center $\left(c_{x}, c_{y}\right)$, skew coefficient and distortion coefficient. The skew coefficient is defined that tangent of the angle between the height axis of camera and CCD cells array, but the coefficient can be ignored since there is generally no skew error in these day of cameras. However, the pinhole camera model is an ideal model and there is practically some image distortion due to the characteristics of lens. The distortion due to the lens is defined as the radial and the tangential distortion coefficient. The ratio of $f_{x}$ and $f_{y}$ is called the aspect ratio which is 1.5 since the ratio of the pixel distance of the width of CCD and the pixel distance of the height is 1.5 in the proposed system. It means that the pixel distance of vertical direction is shorter than the pixel distance of the horizontal direction, if $\mathrm{f}_{\mathrm{y}}$ is larger than $\mathrm{f}_{\mathrm{x}}$.

Pinhole camera model and intrinsic matrix

The correction of distortion due the lens is generally processed after removing the effect of camera internal parameters(Moldovan, 2004). If there is no distortion by the lens, then a point in the physical 3D world is projected to one point on 2D image plane through the pinhole. Then the 2D image plane is called the normalized image plane. If the coordinate of a point in the real world is $\left(\mathrm{X}_{\mathrm{c}}, \mathrm{Y}_{\mathrm{c}}, \mathrm{Z}_{\mathrm{c}}\right)$ and the coordinate of the projected point in the 2D image plane is $\left(x_{n \_}, y_{n \_}\right)$, their relation can be written as the following matrix form where the subscript ' $n \_u$ ' means normalized and undistorted. 


$$
\left[\begin{array}{l}
x_{n_{-} u} \\
y_{n_{-} u}
\end{array}\right]=\left[\begin{array}{l}
X_{c} / Z_{c} \\
Y_{c} / Z_{c}
\end{array}\right]
$$

\section{Experiment procedure and results}

Image of the cloud automatic observing system

As the camera calibration result shows using SWARD tool kit, the intrinsic parameter of camera using is following.

$f_{x}=f_{y}=5,990.57$

$c_{x}=2,558.72 \approx 2,592$ (half of the CCD width)

$c_{y}=1,732.68 \approx 1,728$ (half of the CCD height

$k_{1}=-1.9 \times 10^{-7} \approx 0$

$p_{1}=p_{2} \approx 0$

Equation (3) can be modified by considering above intrinsic parameter as following

$\left[\begin{array}{c}x_{p_{d}} \\ y_{p_{d}} \\ 1\end{array}\right]=\left[\begin{array}{ccc}f_{x} & 0 & c_{x} \\ 0 & f_{y} & c_{y} \\ 0 & 0 & 1\end{array}\right]\left[\begin{array}{c}x_{p} \\ y_{p} \\ 1\end{array}\right]$

Where $f_{x}$ and $f_{y}$ are focal lengths, $x_{p}$ and $y_{p}$ are pixel coordinates of the corrected image. The corrected image is shown in Fig. 5.
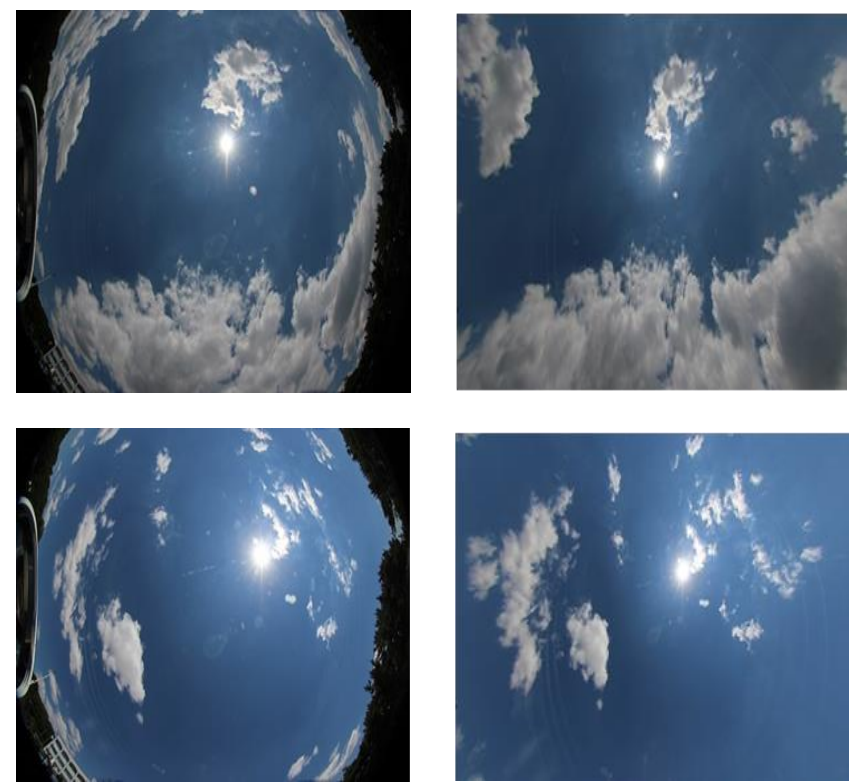

Fig. 5: Corrected Image from Whole Sky Image.

Observing cloud-height

Two cameras are installed with a fixed distance that is $36 \mathrm{~m}$ and axis of two cameras are parallel. This configuration is only good for distance measuring not for remote observing since the binocular disparity is varied with a very wide range.

For the parallel axis stereo camera, the distance (D) from its center to the point $\left(\mathrm{x}_{\mathrm{u}}, \mathrm{y}_{\mathrm{u}}\right)$ can be solved using $(8)$, where $\mathrm{f}$ is the focal distance, $w$ is a half of $\mathrm{L}$, and $\mathrm{plp}_{\mathrm{p}}$ and $\mathrm{p}_{\mathrm{rp}}$ are the distance from the center of CCD to the point on the CCD image as shown in Fig. 6.

$$
D=\sqrt{ }\left(\llbracket\left(w-\left(2 p \_r p w\right) / x_{-} u\right) \rrbracket \wedge 2+\left(2 w f / x_{-} u\right)^{\wedge} 2\right)
$$

$d=p_{-} l p-p \_r p=2 h-2 w f / y_{-} u$

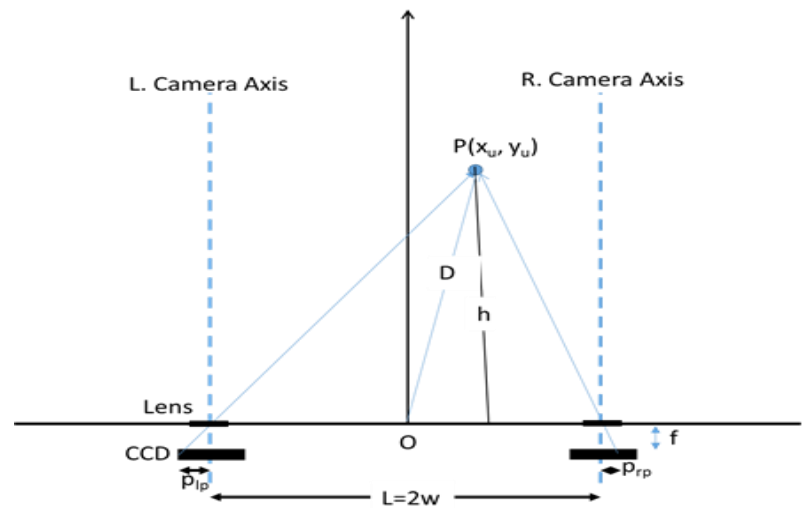

Fig. 6: Pinhole Model of Parallel Axis A Stereo Camera System.

Cloud-height measuring algorithm

After getting a stereo corrected image, BRISK algorithm (Leuteneggeret el, 2011) is applied a pair of images to find key-points or matching points (as shown in Fig. 7) to calculate disparities. Then the cloud-height is measured using the internal parameter of cameras. The number of matching key points is 4,543 which is about 20times of the matching blocks. As a result, BLISK algorithm is more suitable than the block matching algorithm (Tao et el, 2008) to get the height of cloud.

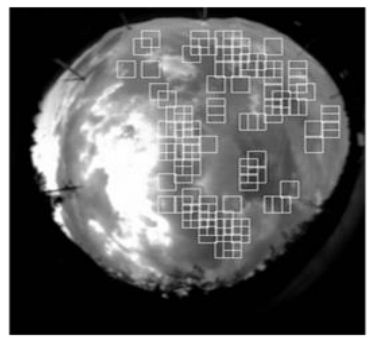

Matching Blocks(Left image)

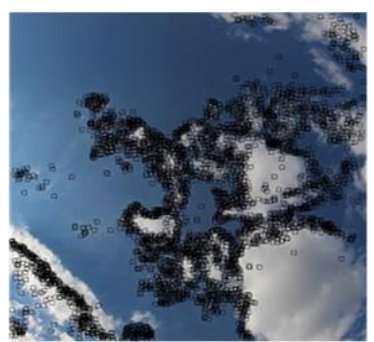

Matching key points(Left image) Matching key points(Right image) Fig. 7: Comparison between Block Algorithm and BLISK Algorithm.

The distance between two cameras is $36 \mathrm{~m}$ for Daejeon local metrological agency. The CCD sensor of camera (Cannon EOS100D) is $22.3(\mathrm{~W}) \times 14.9(\mathrm{H}) \mathrm{mm}$ and its resolution is $5,184 \times 3,456$ pixels. If the observed a cloud height of the ceilometer is $6,000 \mathrm{~m}$ the disparity (d) of the point in the images is obtained as $\mathrm{d}=$ base distance $\mathrm{x}$ focal length/cloud height $=36 \mathrm{~m} \times 8 \times 10^{-3} \mathrm{~m} / 6000 \mathrm{~m}=48 \times 10^{-6} \mathrm{~m}=0.048 \mathrm{~mm}$.

It can be converted to 11.16 pixels for the horizontal direction since 5,184 pixels is in $22.3 \mathrm{~mm}$ (i.e. about $232.5 \mathrm{pixel} / \mathrm{mm}$ ).

\section{Experimental result}

The average of cloud height is about $1,500 \mathrm{~m}$, and rectangular means the matched key points in Fig. 7. The measured disparities is that the average of disparity is 36.7 and the maximum of disparity is 60 pixels. From these disparities, the average of cloud height is $1,822 \mathrm{~m}$ and the minimum of cloud height $1,116 \mathrm{~m}$. 


\section{Conclusion and discussion}

This study is the observing of cloud height and the second stage of the cloud automatic observation project. The first stage was the observing cloud amount and the observing system was developed at that time. To get a stereo image a pair of the observing systems was installed in the Daejeon local metrological agency. The third stage will be observing cloud shapes or cloud types, since cloud shapes are closely related weather and are generally different according to their height. Performing of cloud observing project at present shows in Fig. 8. The dotted line means the first stage.

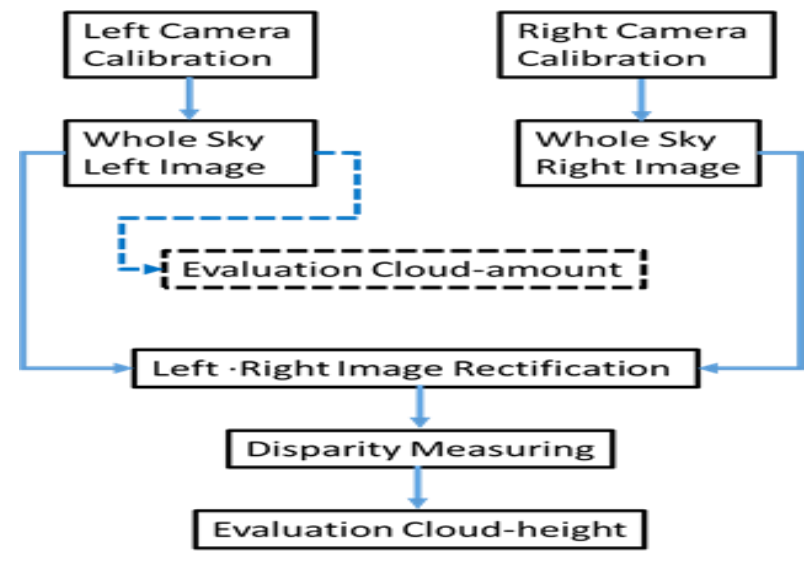

Fig. 8: The Block Diagram of Cloud Automatic Observation.

The procedure to meet the object of this study is show in Fig. 9. The observing result of cloud height is generally acceptable and the algorithm is agreed by the metrological agency. However, the algorithm requires some improvements or upgrade as following. Using BRISK algorithm, key points are higher sensitivity at the cloud edge than the cloud surface. This effect can affect the cloud height since the edge of cloud is actually higher at the edge of fish eye image. Another word, the cloud edge on the edge of a fisheye image is the top of cloud which can reach above $10,000 \mathrm{~m}$.

During the matching processing, the area needed to divide 2 regions such as region between two cameras and outside of each camera, since the sensitivity related to the disparity differs according to the location in the real world.

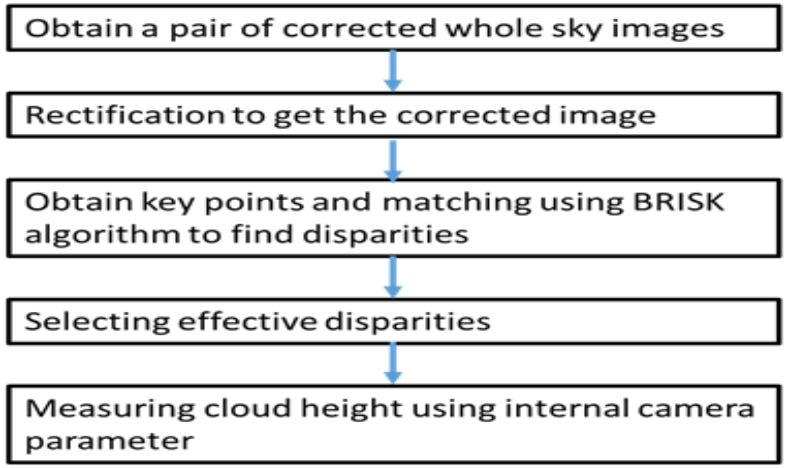

Fig. 9: Measuring Procedure of Cloud Height.

\section{Acknowledgment}

This study is supported by KUT research promotion fund and with university-industry cooperation between AND3D Systems Co.

\section{References}

[1] Allmen, Mark C. \&Kegelmeyer, Philip Jr., 1997, The computation of cloud base height from paired whole-sky imaging cameras, Machine Vision and Applications, 9(4), pp160-165.

[2] Costa-Surós, Montserrat \&Calbó, Josep\& González, Josep-Abel \& Sanchez-Lorenzo, Arturo, 2016, Cloud cover estimation based on ceilometer measurements: a comparison with visual observations, 17th International Conference on Clouds and Precipitation, ICCP2016, Poster.

[3] Kazantzidis, Andreas \&Tzoumanikas, P \&Bais, \&Alkiviadis\& Fotopoulos, Spiros \&Economou, George, 2012, Cloud Detection and Classification with the Use of Whole-Sky Ground-Based Images, Atmospheric Research, 113, pp80-88.

[4] Leutenegger, Stefan, et el, 2011, Binary robust invariant scalable keypoints, International conference on computer vision (ICCV), pp. 2548-2555.

[5] Moldovan, D., Wada, T., 2004, A calibrated pinhole camera model for single viewpoint omnidirectional imaging systems, IEEE International Conference on Image Processing (ICIP), http://ieeexplore.ieee.org/ document/1421738/.

[6] Neto, SylvioMantelli, et el, 2010, The Use of Euclidean Geometric Distance on RGB Color Space for the Classification of Sky and Cloud Patterns, J. of Atmospheric and Oceanic Technology, Vol 27 pp1504-1517.

[7] Sturm, Peter, 2016, Pinhole Camera Model, Computer Vision, Springer, pp 610-613.

[8] Tao, Tangfei, et el, 2008, A fast block matching algorithm for stereo correspondence, IEEE Conference on Cybernetics and Intelligent Systems, pp 38-41

[9] Ying, Xianghua, et el, 2014, Imposing Differential Constraints on Radial Distortion Correction, the 12th Asian Conference on Computer Vision (ACCV'14), pp 384-398.

[10] Ying, Xianghua, et el, 2014, Radial distortion correction from a single image of a planar calibration pattern using convex optimization, IEEE International Conference on Image Processing (ICIP), pp3440-3443. 